\title{
Generalized computational model for high-pressure metal hydrides with variable thermal properties
}

\author{
Mazzucco, Andrea; Rokni, Masoud
}

Published in:

International Journal of Hydrogen Energy

Link to article, DOI:

10.1016/j.ijhydene.2015.03.032

Publication date:

2015

Document Version

Peer reviewed version

Link back to DTU Orbit

Citation (APA):

Mazzucco, A., \& Rokni, M. (2015). Generalized computational model for high-pressure metal hydrides with variable thermal properties. International Journal of Hydrogen Energy, 40(35), 11470-11477.

https://doi.org/10.1016/j.ijhydene.2015.03.032

\section{General rights}

Copyright and moral rights for the publications made accessible in the public portal are retained by the authors and/or other copyright owners and it is a condition of accessing publications that users recognise and abide by the legal requirements associated with these rights.

- Users may download and print one copy of any publication from the public portal for the purpose of private study or research.

- You may not further distribute the material or use it for any profit-making activity or commercial gain

- You may freely distribute the URL identifying the publication in the public portal

If you believe that this document breaches copyright please contact us providing details, and we will remove access to the work immediately and investigate your claim 


\title{
Generalized computational model for high-pressure metal hydrides with variable thermal properties
}

\author{
Andrea Mazzucco, Masoud Rokni* \\ Department of Mechanical Engineering \\ Technical University of Denmark, Nils Koppels Allé 403, DK-2800 Kongens-aLyngby, Denmark \\ andmaz@mek.dtu.dk; $\underline{\text { mr@mek.dtu.dk }}$
}

\begin{abstract}
This study considers a detailed $1 \mathrm{D}$ fueling model applied to the metal hydride system, with $\mathrm{Ti}_{1.1} \mathrm{CrMn}$ as the absorbing alloy, to predict the weight fraction of absorbed hydrogen and solid bed temperature. Dependencies of thermal conductivity and specific heat capacity upon pressure and hydrogen content, respectively, are accounted for, by interpolating experimental data. The effect of variable parameters on the critical metal hydride thickness is investigated and compared to results obtained from a constantparameter analysis. Finally, the discrepancy in the metal hydride thickness value is estimated to be around $10 \%$.
\end{abstract}

Keywords: Hydrogen solid storage, high-pressure metal hydrides, 1D numerical model

\section{Introduction}

Storage systems based on high-pressure metal hydrides (HPMH) have recently been investigated by car manufacturers with the main aim of enhancing volumetric density and reduce filling times for the next generation of hydrogen vehicles [1], [2]. Indeed, the combination of current high-pressure tanks with absorbing alloys having high equilibrium pressures can improve the charge/discharge characteristics and the volume density [3]. Main advantages compared to complex metal hydrides include a faster kinetics, lower absorption temperatures and the capability to release hydrogen at temperatures below $0{ }^{\circ} \mathrm{C}$. Drawbacks of present HPMHs comprise low gravimetric capacities and thermal conductivities [4].

Storage systems based on HPMHs are typically challenging to build and test because of the high pressures involved and the pyrophoric nature of most activated powders [5]. Moreover, the purchase 
cost of these materials makes the storage system relatively expensive even at lab-scale sizes, when different physical layouts have to be investigated. It is therefore extremely important to develop reliable computational models that can predict the system behavior and be applied to full-scale tanks, maintaining a good accuracy and simplicity in the estimation of the main design parameter for the integrated heat exchanger. Different computational models are presented in the literature for HPMHs. However, they all implement metal hydride $(\mathrm{MH})$ parameters independent of the fueling and hydriding conditions [6]-[10].

Constant-parameter models can be developed when a complete property characterization is not available for the selected material or with the goal to lower the complexity of the calculation. Nevertheless, their accuracy must be investigated. In this regard, a detailed computational model in this study is at first compared to the simple approach presented in Ref. [10], the main results of which were used to build two tank prototypes, and consequently, experimental data on specific heat capacity and thermal conductivity are interpolated with dependencies upon hydrogen content and charging pressure respectively, to estimate the critical $\mathrm{MH}$ thickness, as the major design parameter in the heat exchanger design.

\section{Methods}

This study employs an object-oriented language written in Dymola ${ }^{\circledR}$ environment [11] to develop a transient 1D model that is applicable to any absorption/adsorption system, ranging from metal to chemical hydrides. The temperature profile and reaction rate at different locations consist in the main thermal results and stress the importance of the critical metal hydride thickness $\delta$ concept, defined as the maximum distance from the cooling surface at which the reaction is completed below the desired filling time. The evaluation of such a parameter consists in the main result involved in the heat exchanger design process. In this study, a complete absorption is considered to be achieved at $80 \%$ of the $\mathrm{MH}$ saturation value (i.e. $1.5 \mathrm{wt} \%$ for the present material) under a refueling time of $5 \mathrm{~min}$.

Energy and mass balances are coupled with reaction kinetics equations to build the system, enabling the model to be reduced to $0 \mathrm{D}$ and thus, integrated into a more complete refueling system.

To test its accuracy, a constant-parameter approach is at first employed, and the results are compared to those presented in the literature. Then, the effect of variable thermal parameters on the temperature and reaction rate is addressed. Experimental data for properties dependencies upon hydrogen charging pressure and content in the solid bed are interpolated and included in the transient model to evaluate their effect on the critical MH thickness. This analysis provides valuable information on the grade of accuracy with which a constant-parameter model can be used to evaluate the major characteristic length in the heat management system design. 


\subsection{High pressure absorbing alloy}

The analyses carried out in this study consider $\mathrm{Ti}_{1.1} \mathrm{CrMn}$ as the absorbing alloy, to allow for comparisons with results presented in the literature, as discussed in the next section. Interest on this HPMH has aroused for hydrogen storage applications, due to its high capacity per unit volume ( $\sim 50$ $\mathrm{g} / \mathrm{L}$ ) and the capability to perform reasonable hydrogen desorption rates even at temperatures around $-30{ }^{\circ} \mathrm{C}$. The main drawbacks include its high density and low gravimetric capacity $(<2 \mathrm{wt} \%)$, which cause the system to be considerably heavier than the majority of the other on-board storage options, and especially than CHG systems, see Ref. [5]. Furthermore, its low effective conductivity makes the design of the heat exchanger quite challenging, as it significantly limits the heat transfer rates that can be removed from the solid bed, reducing the absorption rates during hydrogen charging. However, it should be mentioned that effective conductivities up to $10 \mathrm{~W} \mathrm{~m}^{-1} \mathrm{~K}^{-1}$ have been obtained with thermal enhancements, according to Ref. [6].

\subsection{Model setup}

For comparison purposes, the domain under study consists in the tank section shown in Fig. 1. The finite temperature difference method is used to discretize such a domain along the $\mathrm{x}$-axis with a discretization step of $1-\mathrm{mm}$. The heat exchanger is assumed to be the U-tube configuration or a tube bank arrangement embedded in the solid bed. The coolant temperature is assumed constant along the tube and thus, only half domain can be considered, as the center line corresponds to an adiabatic boundary.

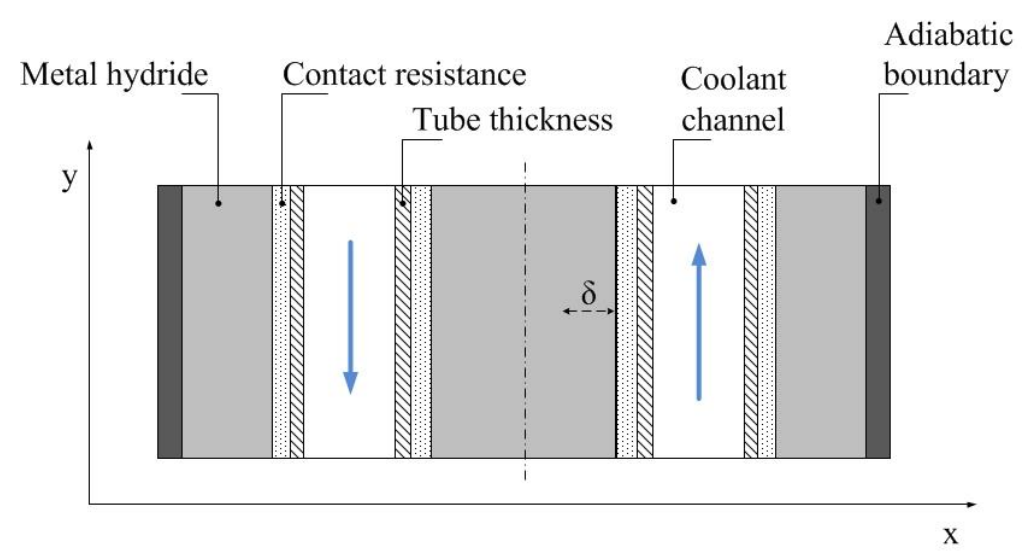

Figure 1. Bed domain considered for discretization, adapted from Ref. [10].

Each location in the solid bed enters into contact with the hydrogen at the same time. Such an assumption is not far from reality when the sintered filters for hydrogen distribution are uniformly placed within the solid bed. Heat transfer is only permitted along the $\mathrm{x}$-axis and the heat generated from the absorption reaction is removed by the coolant that flows in the embedded tubes. The heat is 
transferred from the solid bed to the coolant through the aluminum thickness of the tube and an additional thermal resistance that is modeled to account for the non-perfect contact between the tubes and the absorbing alloy.

The model is accordingly parameterized to allow for comparisons with the results presented in Ref. [10]. However, the parameterization is limited to the information available. For instance, values of $2500 \mathrm{~W} \mathrm{~m}^{-2} \mathrm{~K}^{-1}$ and $2000 \mathrm{~mm}^{2} \mathrm{~K} \mathrm{~W}^{-1}$ for the convection coefficient and the contact resistance between the aluminum tubes and the $\mathrm{MH}$ are considered. Furthermore, the charging pressure is set to 300 bar. The tank pressure rises from 1 bar to the desired value in one minute and is kept constant thereafter to study the temperature profile within the solid bed and the weight fraction of absorbed hydrogen at different distances from the cooling surface.

As shown in the next sub-section the absorption rate is positively influenced by an increase in the operating pressure. Large increases in the charging pressure are needed to influence significantly the hydrogen absorption, because of the logarithmic nature of the reaction rate dependency on the pressure. However, in the model both the charging pressure and its ramp are kept constant, as the effect of these parameters on the hydrogen absorption rate is well known and is out of the scope of this study.

A large porosity value (i.e. $60 \%$ ), that corresponds to a theoretical solid bulk density of $6200 \mathrm{~kg} / \mathrm{m}^{3}$ for $\mathrm{Ti}_{1.1} \mathrm{CrMn}$, results from powder activation, which leads to small particles sizes with sharp edges. This value ensures a moderate compaction that diminishes the risk of further particle deformation during hydrogen absorption and at the same time reduces the contact resistance. In addition, the $60 \%$ porosity allows for the hydride expansion with respect to an expected maximum volume increase by $23 \%$ during hydriding [12]. For this reason, the strain effects that result from the metal hydride swelling are neglected in the model.

The coolant is assumed to be provided from the refueling station along with the hydrogen during charging. As the absorption reaction is the only process requiring cooling, which occurs during fueling, such a strategy allows for reducing the weight and volume of the on-board heat management system by locating the cooling facility in the fueling station, as discussed in Ref. [13]. Another important advantage is the possibility to control the coolant mass flow rate and inlet temperature, which in turns ensures the same cooling conditions at the tank inlet for any vehicle and independently from the particular heat management system integrated in the solid bed.

Experiments have shown that modest flow rates are sufficient to neglect the temperature variation in the coolant during heat absorption. Indeed, in Ref. [14], the results obtained with a constant temperature model show good agreement with the experimental data. In the present study, a value of 45 $\mathrm{L} \mathrm{min}^{-1}$ per tube for the coolant flow rate is needed to provide the convection coefficient mentioned above. Such a value allows neglecting the temperature variation inside the cooling tubes [6]. For this reason, and for comparison purposes, the coolant temperature is here fixed to $0{ }^{\circ} \mathrm{C}$. The coolant properties at this temperature are reported in Table 1.

Table 1. Dexcool ${ }^{\mathrm{TM}}$ properties. 


\begin{tabular}{ll}
\hline Temperature & $\mathbf{2 7 3 ~ K}$ \\
\hline Specific heat capacity, $\mathrm{kJ} \mathrm{kg}^{-1} \mathrm{~K}^{-1}$ & 3.46 \\
\hline Thermal conductivity, $\mathrm{W} \mathrm{m}^{-1} \mathrm{~K}^{-1}$ & 0.415 \\
\hline Density, $\mathrm{kg} \mathrm{m}^{-3}$ & 1060 \\
\hline Kinematic viscosity, $\mathrm{m}^{2} \mathrm{~s}^{-1}$ & $6.80 \times 10^{-6}$ \\
\hline
\end{tabular}

\subsection{Model description}

The fueling model proposed here uses transient mass and energy balances along with a kinetics model to describe the time evolution of solid bed temperature, hydrogen mass flow rate and absorption rate. It is applicable to different MHs by substituting their properties values and modifying, if needed, the kinetic equation. A real gas formulation is implemented for the hydrogen gas properties.

The essential thermal masses involved in the model are the porous medium (being the absorbing alloy) and the hydrogen gas flowing into the system. The latter is partly stored in the absorbed phase and partly in gaseous phase in the pores (being the expansion volume), meaning that the metal hydride system must be treated as a discontinuous medium.

In a typical refueling analysis one is only interested in the hydrogen mass flowing into the tank. The fuel input is thus considered as the accumulation rate in the mass balance. At each moment of the refueling process, the internal hydrogen mass concentration is expressed as,

$$
\dot{m}_{H_{2}}=\varepsilon \cdot V_{M H} \cdot \frac{\partial \rho_{g}}{\partial t}+(1-\varepsilon) \rho_{s} \cdot V_{M H} \frac{\partial w}{\partial t}
$$

where the left-hand-side term (LHS) is the hydrogen mass per unit time flowing into the tank. The first and second terms in the right-hand-side (RHS) represent the rate of accumulated hydrogen in the gas phase and the rate of accumulation in the absorbed phase. The absolute porosity of the metal hydride is expressed with the term $\varepsilon$, while $\rho_{g}, \rho_{s}, w, V_{M H}$ are the gas and solid densities, the weight fraction of hydrogen absorbed in the bed and the MH volume.

The energy balance is applied to the inner tank volume, comprising the MH bed and the coolant, while the pressure vessel is modeled as an adiabatic boundary to allow comparisons with the literature. In porous packed beds, the contact area between gas and solid phases is typically large enough to assume local thermal equilibrium, and hence one single energy equation can be employed to describe the temperature evolution over time for the two phases during hydrogen absorption. This is especially valid when a large value for the porosity is considered, as it occurs in this study. The energy balance is obtained from its general form [15], and reads as:

$$
\frac{\partial H_{s y s t}}{\partial t}=\dot{m}_{H_{2}} h_{i n}+V_{g} \frac{\partial p}{\partial t}+\dot{q}
$$


The RHS of the equation represents the inlet and outlet energy terms related to the hydrogen input mass rate, the compression heat $V_{g} \frac{\partial p}{\partial t}$ and the heat flow exchanged through the solid bed $\dot{q}$.

Considering constant porosity and solid density, the LHS in Eq. (2) is expanded as:

$$
\frac{\partial H_{s y s t}}{\partial t}=\rho_{s} V_{s} \frac{\partial h_{s}}{\partial t}+\rho_{g} V_{g} \frac{\partial h_{g}}{\partial t}+h_{g} V_{g} \frac{\partial \rho_{g}}{\partial t}+\rho_{s} V_{s} w \frac{\partial h_{w}}{\partial t}+\rho_{s} V_{s} h_{w} \frac{\partial w}{\partial t}
$$

where the derivatives involve $h_{s}, h_{g}$ and $h_{w}$, being the specific enthalpy for the absorbing alloy, and hydrogen in the gas and absorbed phases. Eq. (3) in its general form can be applied to absorption and adsorption processes. With a constant heat of absorption and using the continuity equation, it is possible to manipulate the volumetric energy balance (in terms of $V_{M H}$ ) and highlight the single coefficients of $T, p$ and $w$, as:

$$
\begin{aligned}
& \Phi=\left\lfloor(1-\varepsilon) \cdot \rho_{s} w c_{p_{g}}+\rho_{M H} c_{M H}\right\rfloor \\
& \Lambda=\left[(1-\varepsilon)+\left((1-\varepsilon) \cdot \frac{\rho_{s}}{\rho_{g}} w+\varepsilon\right) \cdot\left(1-\alpha_{g} T_{M H}\right)-\varepsilon\right] \\
& \Psi=(1-\varepsilon) \rho_{s} \frac{\Delta H_{a}}{M W_{g}} \\
& \mathrm{I}=\frac{\dot{m}_{H_{2}}}{V_{M H}}\left(h_{i n}-h\right)+q^{\prime \prime \prime}
\end{aligned}
$$

where $\Delta H_{a}, M W_{g}, h_{i n}, h$ and $\alpha_{g}$ are the molar heat of absorption, the $\mathrm{H}_{2}$ molar weight, the specific enthalpy of hydrogen at the inlet, the specific enthalpy of hydrogen in the pores and the isobaric thermal expansion coefficient, respectively. The $\mathrm{H}_{2}$ specific enthalpy in the pores is calculated at the charging pressure $p$ and temperature $T_{M H}$. The volumetric heat rate $q$ "' transferred through the solid bed, has been modeled by means of,

$$
q^{\prime \prime \prime}=\nabla \cdot\left(-k_{M H} \nabla T_{M H}\right)
$$

where $k_{M H}$ is the effective thermal conductivity of the metal hydride. Finally, the solid bed temperature is calculated with Eq. (6):

$$
\Phi \frac{\partial T_{M H}}{\partial t}=-\Lambda \frac{\partial p}{\partial t}-\Psi \frac{\partial w}{\partial t}+\mathrm{I}
$$

The kinetic model used in this study refers to the reaction parameters of $\mathrm{LaNi}_{5}$ and is based on an expression for hydrogen absorption in such a MH derived by Mayer et al. [16] that assumes first order kinetics, as it is often observed in metal hydrides. The reaction parameters of $\mathrm{LaNi}_{5}$ are assumed as 
reasonable engineering estimates for $\mathrm{Ti}_{1.1} \mathrm{CrMn}$, as typically done in the literature. Such an assumption seems to be in good agreement with the results presented in Refs. [14], [17], [18], where an absorption model that uses the present kinetic equation for $\mathrm{Ti}_{1.1} \mathrm{CrMn}$ was validated over experimental data. The reaction rate of hydrogen per unit mass of metal hydride is expressed as,

$$
\frac{\partial w}{\partial t}=C_{a} e^{E_{a} /\left(R T_{M H}\right)} \ln \left(\frac{p}{p^{e q}}\right) \cdot\left(w_{\max }-w\right)
$$

where $p_{e q}$ is the equilibrium pressure of the $\mathrm{MH}, w_{\max }$ is the maximum weight fraction of hydrogen absorbed in the bed, $C_{a}$ is the activation rate constant and $E_{a}$ is the activation energy. The absorption reaction proceeds only when $p>p_{e q}$.

The equilibrium pressure is a function of the metal hydride temperature and is given by the van't Hoff equation:

$$
p^{e q}=p_{0} e^{\frac{\Delta H_{a}}{R T_{M H}}-\frac{\Delta S_{a}}{R}}
$$

where $p_{0}$ and $\Delta S_{a}$ are the ambient pressure and the entropy of reaction respectively.

\subsection{Implementation of variable parameters in the model}

The analyses carried out in this study consider $\mathrm{Ti}_{1.1} \mathrm{CrMn}$ as the absorbing alloy, to allow for comparisons with results presented in the literature, as discussed in the next section. The properties values employed in the constant-parameter model refer to the measurements carried out at the Hydrogen Systems Laboratory (HSL) at Purdue University, see Ref. [10] for the nominal values hereafter considered.

Among these, effective thermal conductivity $\left(k_{M H}\right)$ and specific heat capacity $\left(c_{M H}\right)$ play a major role in the absorption reaction and heat removal. In this study, these parameters are varied as functions of hydrogen charging pressure and content to investigate the effect on the MH critical thickness. This is done by implementing interpolated experimental data in the 1D model.

Measurements have been carried out by Flueckiger et al. with the transient plane source method (TPS) and have been presented in detail in Ref. [19]. The linear interpolation of such data is presented in Fig. 2 and used to discuss the results. Simulations have shown that the overall results are independent of the interpolation method used.

For the activated $\mathrm{Ti}_{1.1} \mathrm{CrMn}$ powder, $k_{M H}$ was measured to be $0.31-0.69 \mathrm{~W} \cdot \mathrm{m}^{-1} \cdot \mathrm{K}^{-1}$ as a function of hydrogen pressure from 2.9 to 253 bar, while no significant dependency on hydrogen concentration was observed. For this reason, the thermal conductivity data are here interpolated as a function of the hydrogen charging pressure, as presented in Fig. 2a. The thermal conductivity increases with the pressure, probably due to the conduction pathways in the gas phase, and a variation was observed in proximity of $170 \mathrm{bar}$, where $k_{M H}$ reached its maximum value in the measured interval. Such a variation falls within the measurement uncertainty and is accounted for in the interpolated data, as its influence in the overall model results is negligible. At the end, it should be mentioned that the measurements 
were carried out at constant temperature and at lower packing densities than what considered in this study. Despite these observations, only the effect on $\delta$ that can be addressed to variable parameters, which have been experimentally measured, is investigated and $k_{M H}$ is assumed not to be influenced by temperature and bed porosity in the current analysis. Nevertheless, it is worth mentioning that in Ref. [12], a theoretical model has addressed the effect of packing density on the effective thermal conductivity of various metal hydrides, including $\mathrm{Ti}_{1.1} \mathrm{CrMn}$. Strategies that aim to augment heat transfer in the solid bed by increasing the packing density are expected to be more effective than enhancing the intrinsic conductivity of MH particles.

The specific heat experienced a dramatic increase, up to $1050 \mathrm{~J} \cdot \mathrm{kg}^{-1} \cdot \mathrm{K}^{-1}$ at a charging pressure around 170 bar, as a result of the transition to the $\beta$-phase lattice structure and resulting phonon transport at the hydrogen occupied interstitials of the $\mathrm{MH}$ lattice. By relating the charging pressure and temperature to the hydrogen absorption, it was observed that such an abrupt increase is a function of the reaction progress. For this reason, $c_{M H}$ data are interpolated as a function of the reaction progress variable, which is defined as,

$$
F=\frac{w}{w_{\max }}
$$

where $w$ and $w_{\max }$ are the weight fraction of hydrogen in the solid bed and its maximum value reached at saturation conditions respectively. The dependency of $c_{M H}$ upon the reaction progress is shown in Fig. 2b.

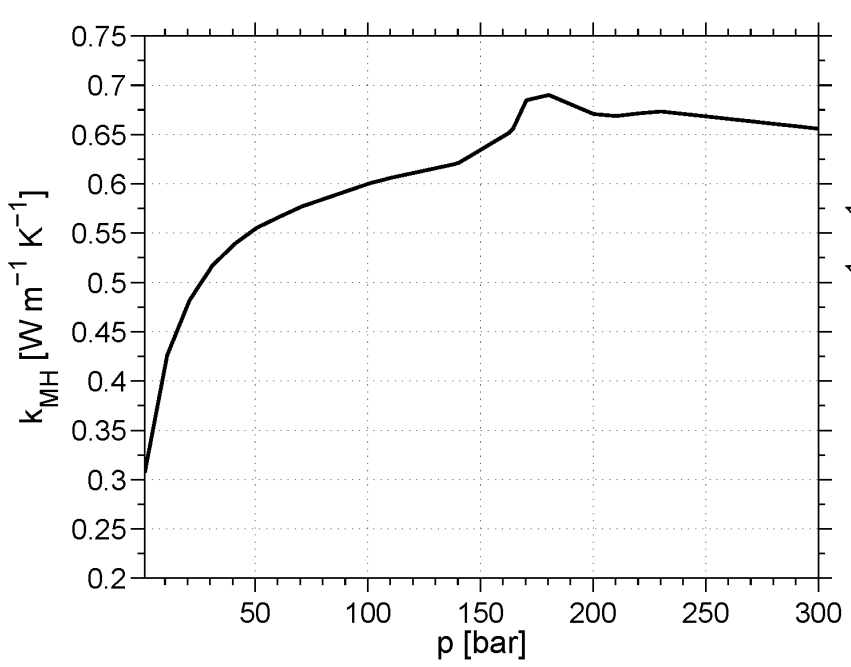

(a)

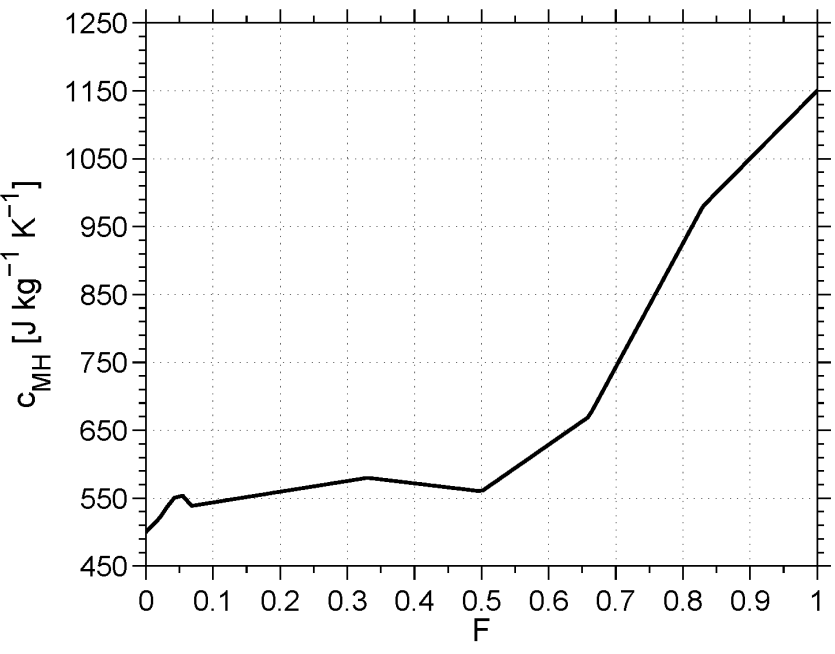

(b)

Figure 2. Interpolated thermal conductivity over charging hydrogen pressure (a) and specific heat capacity over reaction progress (b). 


\section{Results}

\subsection{Constant-parameter model.}

At first the model is simulated with constant $k_{M H}$ and $c_{M H}$, the nominal values of which are $1 \mathrm{~W} \mathrm{~m}^{-}$ ${ }^{1} \mathrm{~K}^{-1}$ and $500 \mathrm{~J} \mathrm{~kg}^{-1} \mathrm{~K}^{-1}$ respectively. The comparison with the results obtained with a simple heatdiffusion-equation model presented in Ref. [10] is shown in Fig. 3 for the volumetric heat rate of generation, the reaction progress $F$, and the temperature distribution in the solid bed at different locations from the cooling element.

The volumetric heat rate of generation is presented in Fig. 3a. It takes into account the heat of pressurization and the reaction heat in the solid bed. The reaction heat is a function of the absorption rate, while the heat of pressurization is only function of the pressure ramp (and the porosity), which is positive and constant until the 300 bar condition is reached. For this reason, the heat of generation starts at a value different from zero and remains constant until the absorption reaction takes place. A good agreement is obtained with the results in the literature. However, the maximum relative difference increases with the distance from $2.6 \%$ at $0 \mathrm{~mm}$ to around $25 \%$ at $7.5 \mathrm{~mm}$. The solid in direct contact with the cooling element experiences the greatest heat generation rate, of about $3.73 \mathrm{MW}$, which corresponds to the highest cooling rate. The peak decreases with the distance to the cooling surface and remains nearly constant for the solid at a distance beyond $8 \mathrm{~mm}$, as the total thermal resistance is large enough to control the heat removal and thus, the absorption process.

Similar conclusions can be drawn from Fig. $3 \mathrm{~b}$ where the profile temperature in the reaction bed is shown. However, the maximum relative difference $(2 \%)$ occurs at the end of pressurization for the solid at $0 \mathrm{~mm}$ and corresponds to a discrepancy of $6.5 \mathrm{~K}$ in the results.

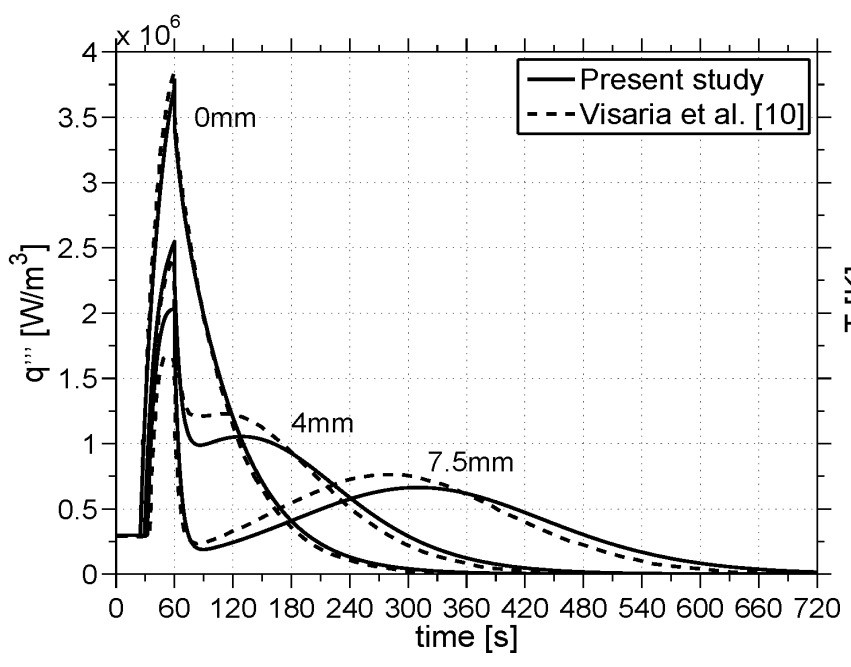

(a)

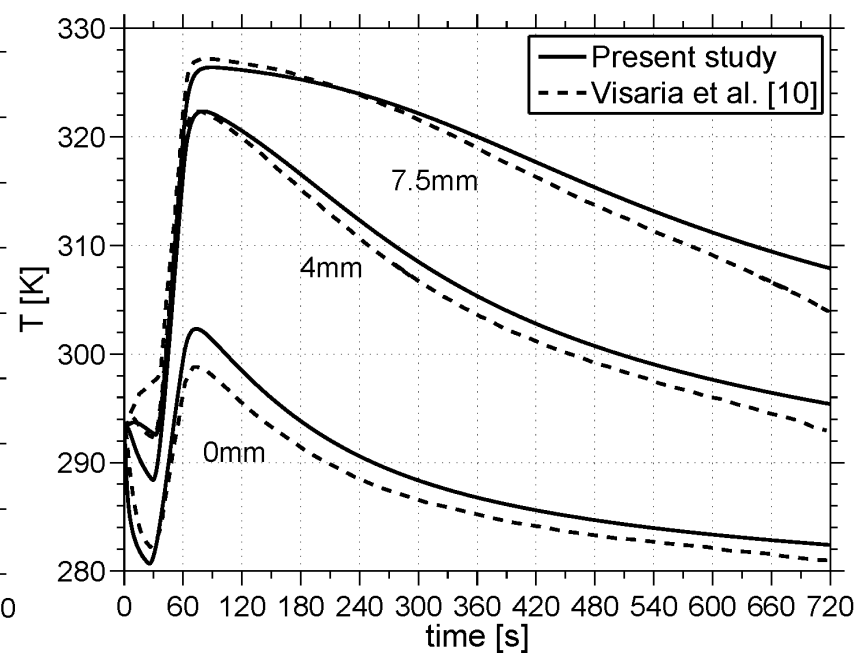

(b) 


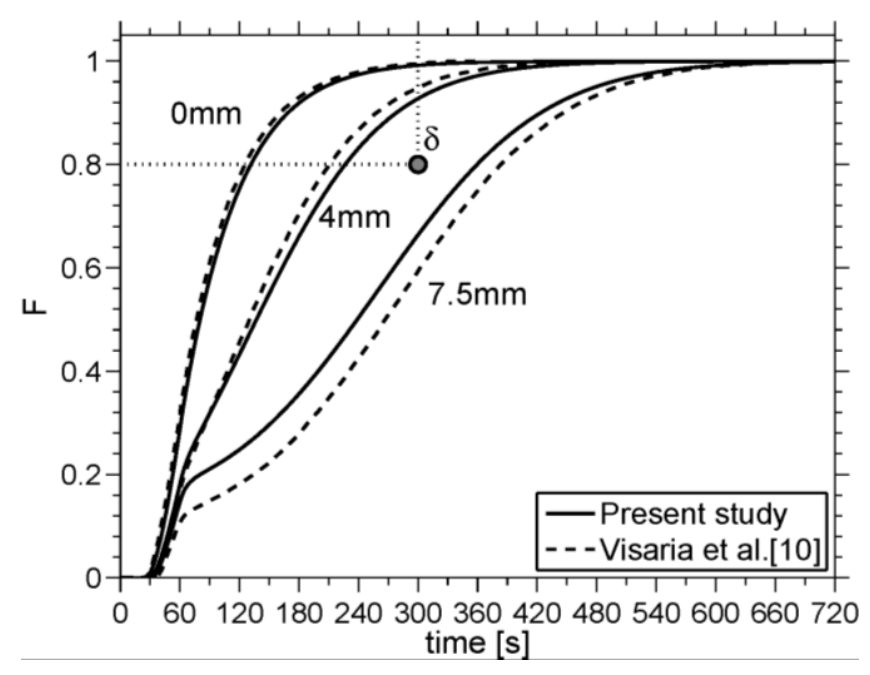

(c)

Figure 3. Comparison of results for heat generation rate per unit volume (a); bed temperature (b); reaction progress $F(\mathrm{c})$ at different locations, with $\delta$ being the maximum distance from the cooling surface at which the reaction is completed below the desired filling time of $5 \mathrm{~min}$.

From Fig. $3 \mathrm{c}$ one can see that the disparity in the reaction progress value between current and literature results increases with the distance from the cooling element. The maximum relative difference in results ranges from nearly $0 \%$ for the solid in contact with the tube, to $28 \%$ at $7.5 \mathrm{~mm}$. The reaction starts earlier for the solid closer to the coolant. For instance, at a location of $7.5 \mathrm{~mm}$, the reaction does not start before 32 seconds, while the solid in direct contact with the tube starts reacting at 25 seconds. For the latter, no inflection in the reaction rate can be detected and the absorption process at this location is kinetics-limited. The grey dotted lines identify the desired region of operation for the entire solid bed. This corresponds to the zone where a complete reaction is achieved by the time of $5 \mathrm{~min}$. The critical thickness coincides to the limit of such a region and is evaluated to be about 5 $\mathrm{mm}$ in accordance with the results reported in the literature.

The fact that the agreement in results generally decreases for locations further from the tube, indicates that the discrepancy in the models is mainly due to thermal differences, the effect of which is more significant at larger solid thicknesses. These can be attributed to the different nature of the models, gas equations and to the non-perfect parameterization.

\subsection{Variable-parameter model}

Measurements for $k_{M H}$ and $c_{M H}$ showed lower and greater values, respectively, than in the nominal case. As a result, the temperatures in the solid bed are expected to increase for the combination of larger thermal resistances and inertia, that are attributed to the reduction in the conductivity and 
increase in heat capacity. This can be seen in Fig. 4a, where the solid bed temperatures are plotted at different distances from the cooling surface for constant and variable $\mathrm{MH}$ parameters.

When variable parameters are considered, higher temperatures occur before and after pressurization. In light of Figs. $2 \mathrm{a}$ and 2b, a lower thermal conductivity is experienced in the solid bed at the beginning of the hydrogen charging, when the absorption reaction has yet to start and the heat capacity is constant and equal to its nominal value. In $60 \mathrm{~s}$ the filling process is completed and $k_{M H}$ is close to its maximum value, settling at about $0.65 \mathrm{~W} \mathrm{~m}^{-1} \mathrm{~K}^{-1}$, which is still far from the unitary value used in the constant parameter model. When the absorption reaction starts, the MH heat capacity increases slowly as long as the reaction progress is below 0.5, while it experiences a dramatic augmentation as the reaction proceeds further. As a result, these properties variation provide larger thermal resistances, decreasing the cooling rates, and greater thermal inertias that reduce the speed of temperature response to the cooling rate. The increase in temperature is less significant for thicknesses above $12 \mathrm{~mm}$, as at this location the temperature reaches the adiabatic value of $55^{\circ} \mathrm{C}$ at the end of pressurization and cannot increase further.

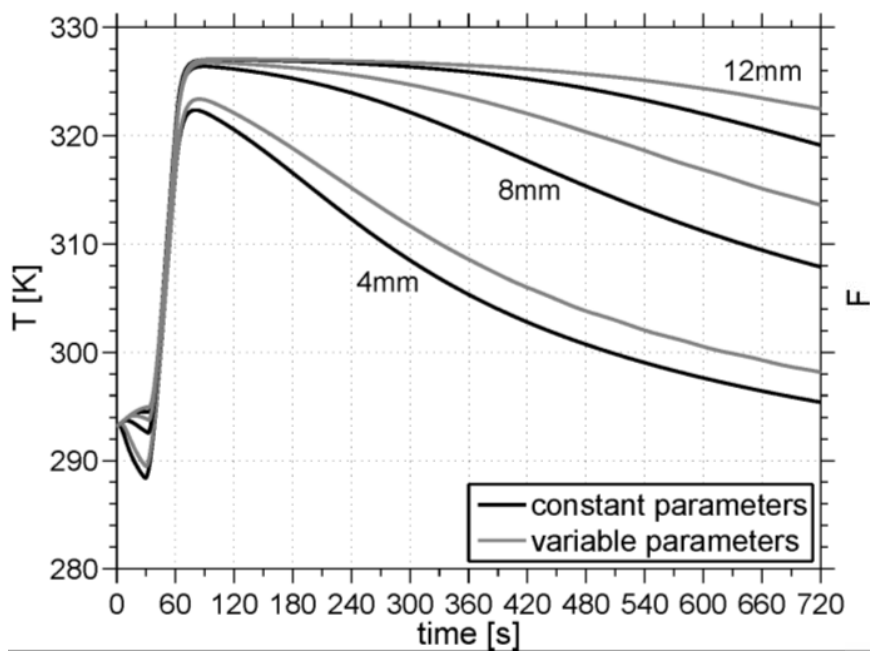

(a)

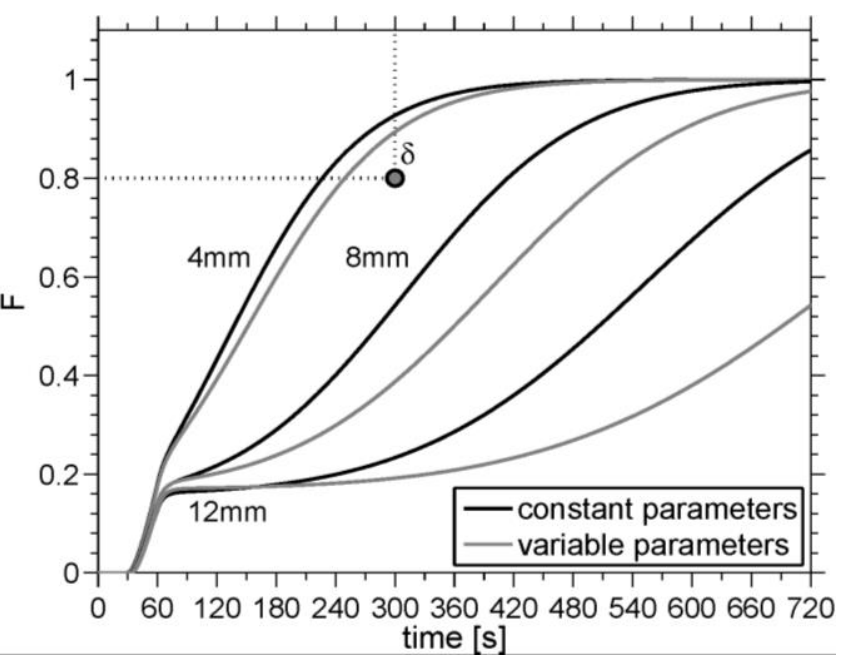

(b)

Figure 4. Effect of variable parameters on bed temperature (a) and reaction progress of hydrogen absorbed (b) in the bed, with $\delta$ being the maximum distance from the cooling surface at which the reaction is completed below the desired filling time of 5 min.

The effect of temperature on the reaction rate depends on the total thermal resistance between the solid powder and the coolant; see Fig. $4 \mathrm{~b}$ where the reaction progress over time is presented. At locations near the cooling surface, the reaction is kinetics-limited and a few degrees increase in the temperature do not affect much the reaction rate, as the total thermal resistance is low enough to enable large cooling rates. The contrary occurs at further distances, at which the reaction is heat-transfer- 
limited. The increase in the thermal inertia and resistance makes the new $F$ curves shift towards longer filling times. As a result, the new value of $\delta$ is reached for the solid powder at a location close to 4.5 $\mathrm{mm}$.

Finally, with variable $\mathrm{MH}$ parameters, the critical thickness reduces by nearly $0.5 \mathrm{~mm}$, which corresponds to $10 \%$ of the value estimated from the constant-parameter model. Thus, for $\mathrm{Ti}_{1.1} \mathrm{CrMn}$, the $\delta$ value is not significantly affected by the implementation of variable thermal parameters. The effect is expected to be further reduced in practice, because of the positive influence of temperature increase on the enhancement of $k_{M H}$ during exothermic absorption, which was not considered in the measurements.

\subsection{Influence of conductivity and specific heat capacity}

The effect of $k_{M H}$ and $c_{M H}$ is separately investigated to study whether a predominant influence on the metal hydride thickness can be addressed to one of such parameters. The model is modified to take into account only the dependency of the thermal conductivity upon the pressure, while the specific heat capacity is kept constant and equal to its nominal value. The former scenario is compared with the variable parameters case in Fig. 5 with respect to the reaction progress.

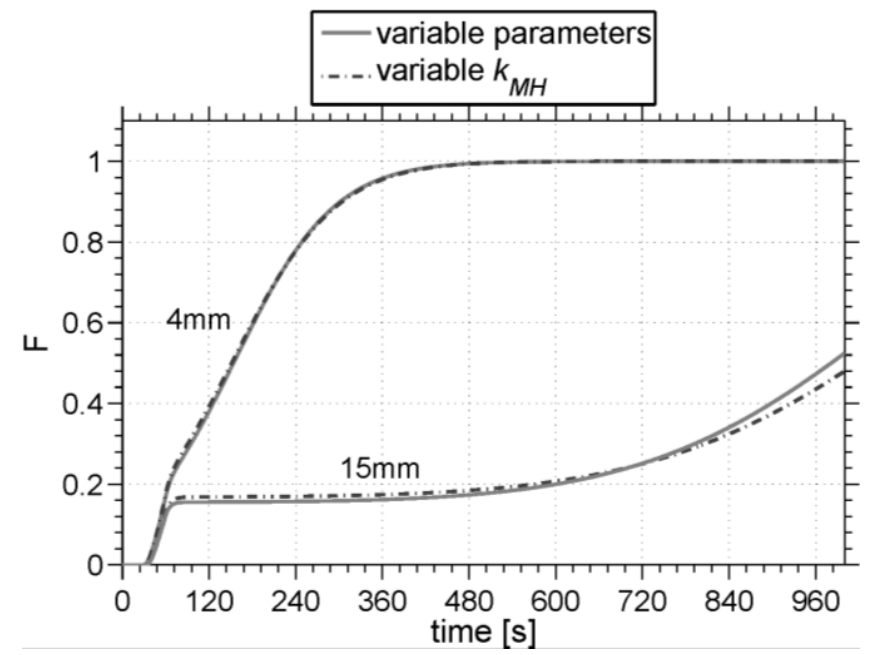

Figure 5. Reaction progress of absorbed hydrogen for variable parameters and variable $k_{M H}$ only.

Two locations representative of small and large solid thicknesses are selected. At $4 \mathrm{~mm}$ the curves are nearly coincident. When a discrepancy is observed after pressurization, as at a distance of $15 \mathrm{~mm}$, the effect of the $c_{M H}$ is a decrease in the reaction rate due to the higher temperature related to larger thermal inertia. However, such influence seems to be negligible on the weight fraction of absorbed hydrogen. It follows that, the disparity in results observed in Fig. 4 should be addressed mainly to the lower values of thermal conductivities. The main reason is that $c_{M H}$ varies with $F$ more rapidly at locations near the cooling surface, where the metal hydride experiences intensive cooling rates and is dominated by kinetics. Indeed, the significant variation in the heat capacity that occurs between $50 \%$ 
and $100 \%$ of the reaction progress (see Fig. 2b) is realized at shorter times for the powder in proximity to the cooling tube, where the temperature drops at a faster rate despite the increase of thermal inertia. As a result, its effect on the reaction rate is negligible. On the contrary, at larger distances, the large total thermal resistance strongly reduces the cooling rates, preventing the solid to achieve reaction rates high enough to trigger a significant variation in the $c_{M H}$ value. At $15 \mathrm{~mm}$ and for a reaction time below $500 \mathrm{~s}, F$ is 0.2 and the $c_{M H}$ settles on a value around $550 \mathrm{~J} \mathrm{~kg}^{-1} \mathrm{~K}^{-1}$, which is very close to its nominal value.

\section{Conclusion}

Thermal conductivity and specific heat capacity dependencies upon pressure and hydrogen content respectively, seem to have a small effect on the calculation of the critical metal hydride thickness for $\mathrm{Ti}_{1.1} \mathrm{CrMn}$. A constant-parameter 1D model describes the reaction rates and temperature distribution in the solid bed with a good accuracy for the purpose of $\delta$ estimation. The difference in results for the critical metal hydride thickness was calculated to be $10 \%$. This difference is to be addressed mainly to the lower thermal conductivity, while the specific heat capacity does not play a significant role.

The generalized constant-parameter model provides results that are consistent within the literature and unlike previous models; it is developed so that it can be easily reduced to OD for the purpose of integration in a complete refueling system. This gives the opportunity in future studies, to identify the optima refueling strategies for HPMH tanks, whose heat management system is designed in respect of the $\delta$ criterion.

\section{Acknowledgements}

The authors acknowledge the Danish Energy Agency for financial support and all the members of the Hyfill-Fast International Research Project for their collaboration. The authors also acknowledge Dr. Pourpoint and his team, at Purdue University, for the helpful insights and the courtesy of sharing their experimental data.

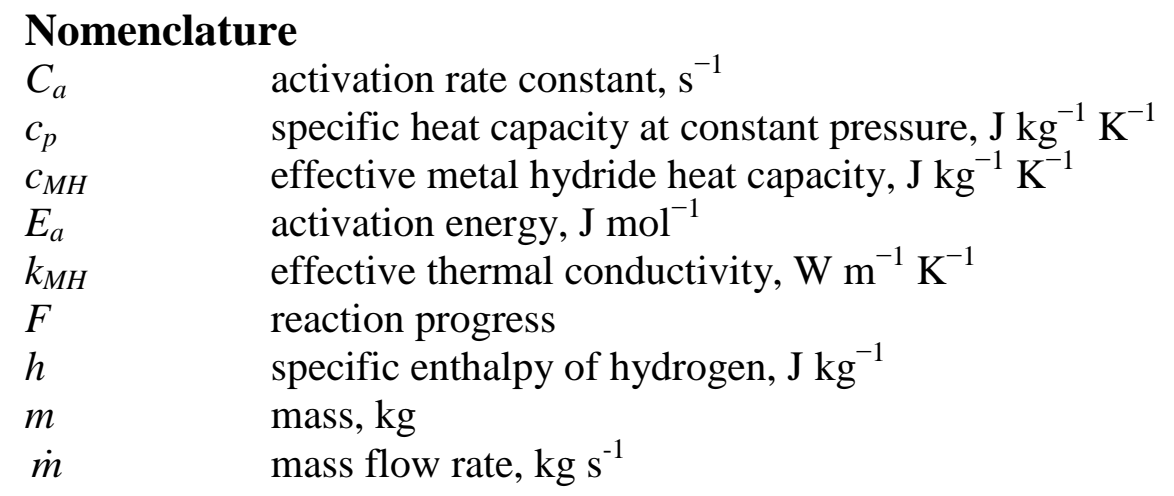




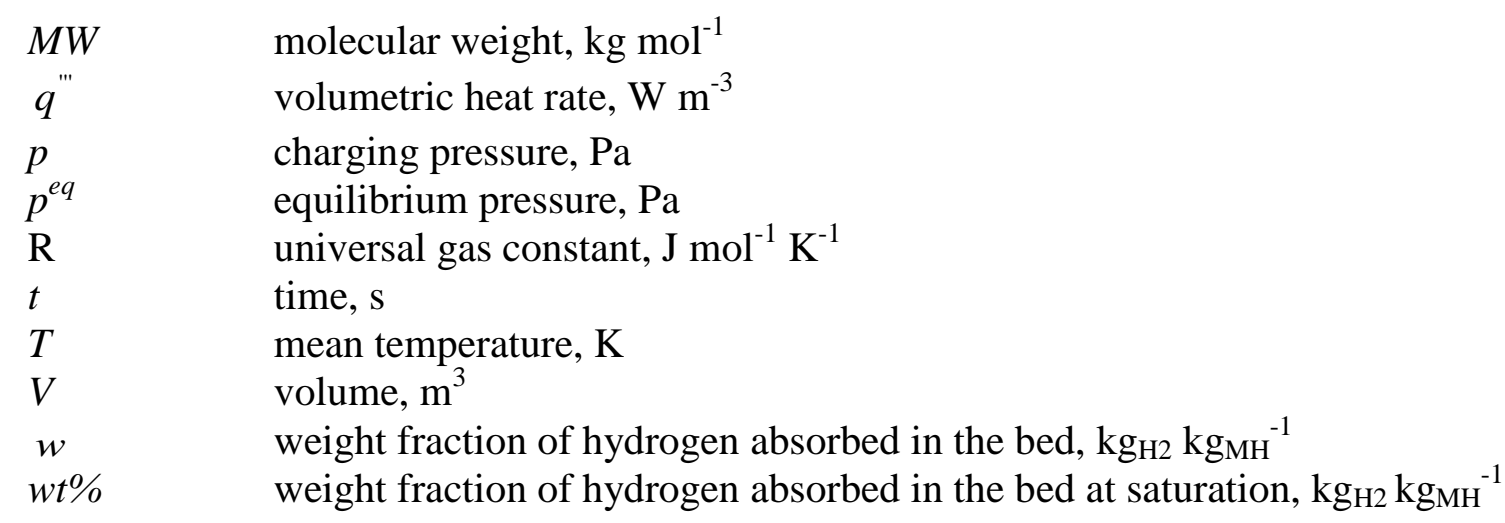

\section{Greek symbols}

$\alpha$

$\Delta H_{a}$

isobaric thermal expansivity, $\mathrm{K}^{-1}$

$\Delta S_{a}$

$\delta$

enthalpy of absorption, $\mathrm{J} \mathrm{mol}^{-1}$

entropy of absorption, $\mathrm{J} \mathrm{K}^{-1} \mathrm{~mol}^{-1}$

$\varepsilon$

I

$\Lambda$

critical metal hydride thickness, $\mathrm{mm}$

void fraction of the metal hydride

constant term in the energy balance, $\mathrm{W} \mathrm{m}^{-3}$

$\rho$

coefficient of the time derivative of pressure

$\rho \quad$ density, $\mathrm{kg} \mathrm{m}^{-3}$

$\Phi \quad$ coefficient of the time derivative of bed temperature, $\mathrm{J} \mathrm{m}^{-3} \mathrm{~K}^{-1}$

$\Psi \quad$ coefficient of the time derivative of weight fraction, $\mathrm{J} \mathrm{m}^{-3}$

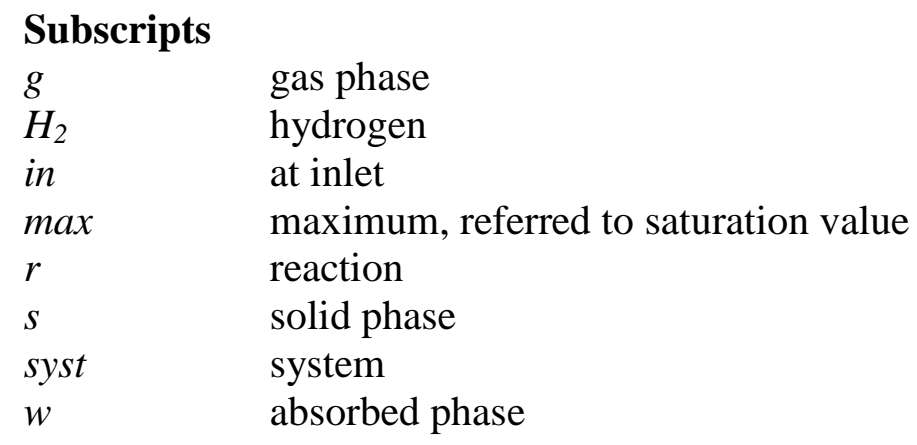

$\begin{array}{ll}\text { Abbreviations } \\ \text { CHG } & \text { Compressed Hydrogen Gas } \\ \text { HPMH } & \text { High Pressure Metal Hydride } \\ \text { LHS } & \text { Left Hand Side } \\ \text { MH } & \text { Metal Hydride } \\ \text { RHS } & \text { Right Hand Side }\end{array}$




\section{References}

[1] D. Mori, K. Hirose, N. Haraikawa, T. Takiguchi, T. Shinozawa, T. Matsunaga, K. Toh, K. Fujita, A. Kumano, and H. Kubo, "High-pressure Metal Hydride Tank for Fuel Cell Vehicles," pp. 1-5, Jul. 2007.

[2] K. Toh, H. Kubo, Y. Isogai, D. Mori, K. Hirose, and N. Kobayashi, "Thermal Analysis of HighPressure Metal Hydride Tank for Automotive Application," MRS Symp. Proc., vol. 927, pp. 0927-EE01-07, Feb. 2011.

[3] N. Takeichi, H. Senoh, and N. Kuriyama, "Application of hydrogen storage alloy at high pressure over $30 \mathrm{MPa}$," Meet. Abstr. - 2004 Jt. Int. Meet. - 206th Meet. Electrochem. Soc., p. $549,2004$.

[4] B. Sakintuna, F. Lamaridarkrim, and M. Hirscher, "Metal hydride materials for solid hydrogen storage: A review云,” Int. J. Hydrogen Energy, vol. 32, no. 9, pp. 1121-1140, Jun. 2007.

[5] Y. Kojima, Y. Kawai, S. Towata, T. Matsunaga, T. Shinozawa, and M. Kimbara, "Development of metal hydride with high dissociation pressure," J. Alloys Compd., vol. 419, no. 1-2, pp. 256261, Aug. 2006.

[6] M. Raju, J. P. Ortmann, and S. Kumar, "System simulation model for high-pressure metal hydride hydrogen storage systems," Int. J. Hydrogen Energy, vol. 35, no. 16, pp. 8742-8754, Aug. 2010.

[7] Y. Zheng, V. Velagapudi, T. Pourpoint, S. T. Fisher, I. Mudawar, and J. P. Gore, "Thermal management analysis of on-board high-pressure metal hydride systems," in Proceedings of IMCE 2006 - ASME International Mechanical Engineering Congress and Exposition, 2006, pp. $1-8$.

[8] K. C. Smith, Y. Zheng, T. S. Fisher, T. L. Pourpoint, and I. Mudawar, "Heat transfer in metal hydride systems," J. Enhanc. Heat Transf., vol. 16, no. 2, pp. 189-203, 2009.

[9] T. L. Pourpoint, V. Velagapudi, I. Mudawar, Y. Zheng, and T. S. Fisher, "Active cooling of a metal hydride system for hydrogen storage," Int. J. Heat Mass Transf., vol. 53, no. 7-8, pp. 1326-1332, Mar. 2010.

[10] M. Visaria, I. Mudawar, T. Pourpoint, and S. Kumar, "Study of heat transfer and kinetics parameters influencing the design of heat exchangers for hydrogen storage in high-pressure metal hydrides," Int. J. Heat Mass Transf., vol. 53, no. 9-10, pp. 2229-2239, Apr. 2010.

[11] “Dymola, Dynamic Modeling Laboratory - User's Manual,” Dynasim AB. 
[12] K. C. Smith and T. S. Fisher, "Models for metal hydride particle shape, packing, and heat transfer," Int. J. Hydrogen Energy, vol. 37, no. 18, pp. 13417-13428, 2012.

[13] A. Mazzucco, M. Dornheim, M. Sloth, T. R. Jensen, J. O. Jensen, and M. Rokni, "Bed geometries, fueling strategies and optimization of heat exchanger designs in metal hydride storage systems for automotive applications: A review," Int. J. Hydrogen Energy, vol. 39, no. 30, pp. 17054-17074, Oct. 2014.

[14] M. Visaria and I. Mudawar, "Experimental investigation and theoretical modeling of dehydriding process in high-pressure metal hydride hydrogen storage systems," Int. J. Hydrogen Energy, vol. 37, no. 7, pp. 5735-5749, Apr. 2012.

[15] R. B. Bird, W. E. Stewart, and E. N. Lightfoot, Transport phenomena, 2nd ed. New York (USA): John Wiley \& Sons, 2002, p. 335.

[16] U. Mayer, M. Groll, and W. Supper, "Heat and mass transfer in metal hydride reaction beds: experimental and theoretical results," J. Less Common Met., vol. 131, pp. 235-244, 1987.

[17] M. Visaria, I. Mudawar, and T. Pourpoint, "Enhanced heat exchanger design for hydrogen storage using high-pressure metal hydride: Part 1. Design methodology and computational results," Int. J. Heat Mass Transf., vol. 54, no. 1-3, pp. 413-423, Jan. 2011.

[18] M. Visaria, I. Mudawar, and T. Pourpoint, "Enhanced heat exchanger design for hydrogen storage using high-pressure metal hydride - Part 2. Experimental results," Int. J. Heat Mass Transf., vol. 54, no. 1-3, pp. 424-432, Jan. 2011.

[19] S. Flueckiger, T. Voskuilen, T. Pourpoint, T. S. Fisher, and Y. Zheng, "In situ characterization of metal hydride thermal transport properties," Int. J. Hydrogen Energy, vol. 35, no. 2, pp. 614621, Jan. 2010. 